\title{
Belle: Charmless hadronic B meson decays with Belle
}

\author{
Min-Zu Wang ${ }^{* \dagger}$ \\ National Taiwan University, Taiwan \\ E-mail: mwang@phys.ntu.edu.tw
}

Recent results of charmless $B$ decays from Belle are reported. These include the two-body decays of $\eta h, \omega h$, and $\eta^{\prime} h$, and $\eta h h$ three-body decay, where $h$ stands for kaon or pion. The charge asymmetries of high statistics modes are also reported. This study is based on a $357 \mathrm{fb}^{-1}$ data sample, consisting of 386 million $B \bar{B}$ pairs, collected by the Belle detector at the KEKB asymmetric energy $e^{+} e^{-}(3.5$ on $8 \mathrm{GeV})$ collider.

International Europhysics Conference on High Energy Physics

July 21 st - 27th 2005

Lisboa, Portugal

*Speaker.

${ }^{\dagger}$ on behalf of the Belle Collatoration 


\section{Introduction}

Charmless hadronic $B$ decays provide a rich ground to study the dynamics of $B$ meson and $C P$ violation phenomenon. In the Standard Model, direct $C P$ violation can arise in particle decays from the interference of two or more amplitudes with both strong phases and weak phases being different. Many charmless hadronic $B$ meson decays contain both tree and penguin amplitudes and provide a good sample for direct $C P$ violation searches. Since the $B^{-}$meson contains a $b$ quark, we adopt the convention that the direct $C P$ asymmetry in a $B \rightarrow f$ decay is defined as

$$
A_{C P}=\frac{\Gamma\left(B^{-} \rightarrow f^{-}\right)-\Gamma\left(B^{+} \rightarrow f^{+}\right)}{\Gamma\left(B^{-} \rightarrow f^{-}\right)+\Gamma\left(B^{+} \rightarrow f^{+}\right)}
$$

It has been suggested that the direct $C P$ violation could be large in $B^{-} \rightarrow \eta \pi^{-1}$, and $B^{-} \rightarrow$ $\eta K^{-}$decays [1]. Previous measurements do not have enough statistics to determine this effect. The $B \rightarrow \eta^{\prime} X_{S}$ decay is still an unsolved puzzle for many theorists. It provides the largest branching fractions among the charmless hadronic decays of $B$ mesons. The observed branching fractions of $B \rightarrow \eta^{\prime} K$ [2] are larger than the expectation from generalized factorization approach. It is speculated that $\mathrm{SU}(3)$-singlet couplings unique to $\eta^{\prime}$ meson or new physics contribute to the amplitude. The two-body $B$ decays with a vector meson $\omega$ and a pseudoscalar particle $h$ [3] proceed through $b \rightarrow s$ penguin diagrams and spectator $b \rightarrow u$ diagrams. Theoretical expectations from QCD factorization approaches suggest that the branching fractions of charged $B$ decays are $\sim(3-8) 10^{-6}$ and the $\omega K^{-}$branching fraction is smaller than the $\omega \pi^{-}$. The decay $B^{0} \rightarrow \omega \pi^{0}$ is color suppressed while the neutral $B$ decays to $\omega K$ is expected to have slightly smaller branching fraction than the charged $B$ decays.

We present a study of the above two-body decays, namely $\eta h, \omega h$, and $\eta^{\prime} h$ of $B$ mesons, where $h$ stands for kaon or pion. It is based on a $357 \mathrm{fb}^{-1}$ data sample, consisting of 386 million $B \bar{B}$ pairs, collected by the Belle [5] detector at the KEKB asymmetric energy $e^{+} e^{-}(3.5$ on $8 \mathrm{GeV})$ collider.

There are interests for $B^{0} \rightarrow a_{0}^{-} h^{+}$and the observation of these decay modes will provide information both on $B$ meson decays to scalar mesons and on the nature of the $a_{0}^{-}$meson. We also use the same data sample to study the three-body decays, $B^{0} \rightarrow \eta h^{+} h^{-}$[4], which contains the intermediate decay process of $B^{0} \rightarrow a_{0}^{-} h^{+}$.

\section{Analysis procedure}

The challenge of observing rare $B$ decay processes is to fish out a few signal events from background events. Since the center-of-mass energy is set to match the $\Upsilon(4 S)$ resonance and $\Upsilon(4 S)$ decays into a $B \bar{B}$ pair, we can use the following two kinematic variables to identify the reconstructed $B$ meson candidates: the beam-energy constraint mass, $M_{b c}=\sqrt{E_{b e a m}^{2}-p_{B}^{2}}$, and the energy difference, $\Delta E=E_{B}-E_{\text {beam }}$, where $E_{\text {beam }}, p_{B}$ and $E_{B}$ are the beam energy, the momentum and energy of the reconstructed $B$ meson in the rest frame of $\Upsilon(4 S)$, respectively. The resolution of $M_{b c}$ is about $3 \mathrm{MeV} / \mathrm{c}^{2}$ which is due to the spread of the beam energy. Typically, the resolution of $\Delta E$ is

\footnotetext{
${ }^{1}$ Throughout this report, inclusion of charge conjugate mode is always implied unless otherwise stated.
} 
about $10 \mathrm{MeV}$ for final states with charged particles only. It has a wider spread and a low energy tail in the $\Delta E$ spectrum for modes with $\pi^{0}(\gamma)$. The signal shape and its efficiency is determined by GEANT [6] based Monte Carlo (MC) simulation.

The generic $B$ decay is mainly via the $b \rightarrow c$ transition which normally has more final state particles than those of the rare decay modes reported here, thus the background from generic $\mathrm{B}$ decays is much less than that from the continuum process. Similar $B$ decay processes close to the target mode should be checked carefully one by one because they might feed into the signal region. One important feature is a $50 \mathrm{MeV}$ shift in the $\Delta E$ spectrum due to $\pi-K$ misidentification. For the continuum events, they have quite different event topology (more back-to-back or jet-like) than that of $B \bar{B}$ events (more spherical) in the $\Upsilon(4 S)$ frame. We can select some shape variables to form a Fisher discriminant in order to reject the continuum background. After the optimization of selection cuts, events in the candidate $M_{b c}-\Delta E$ region are used for yield determination. This is done by un-binned likelihood fit. If there is no evidence of signal events, one can use the fit results to estimate the expected background, and compare this with the observed number of events in the signal region in order to set the upper limit on the yield at the $90 \%$ confidence level [7].

\section{Results}

The results of this study are summarized in Table 1. A typical plot for checking the $A_{C P}$ of $B^{-} \rightarrow \eta K^{-}$is shown in Fig. 1. We are looking forward to exciting news from $B$ factories with more data in the near future.

Table 1: Measured branching fractions ( $\mathscr{B}$ ), the $90 \%$ confidence level upper limits (UL) on the branching fractions and $A_{C P}$ 's for different $B$ decays modes. Note that the branching fractions related to $a_{0}^{-}$are the products of branching fractions for $B$ decays and $a_{0}^{-} \rightarrow \eta \pi^{-}$.

\begin{tabular}{|l|c|c|c}
\hline Mode & $\mathscr{B}_{r}\left(\times 10^{-6}\right)$ & $\mathrm{UL}\left(\times 10^{-6}\right)$ & $A_{C P}$ \\
\hline$\eta K^{-}$ & $2.2 \pm 0.4 \pm 0.1$ & - & $-0.55 \pm 0.19 \pm 0.04$ \\
$\eta \pi^{-}$ & $3.9 \pm 0.5 \pm 0.2$ & - & $-0.10 \pm 0.11 \pm 0.02$ \\
$\eta K^{0}$ & $0.9 \pm 0.6 \pm 0.1$ & $<1.9$ & - \\
$\eta^{\prime} K^{-}$ & $68.6 \pm 2.1 \pm 3.6$ & - & $0.029 \pm 0.028 \pm 0.02$ \\
$\eta^{\prime} \pi^{-}$ & $1.73_{-0.63}^{+0.69} \pm 0.12$ & - & $0.15_{-0.38+0.02}^{+0.39+0.02}$ \\
$\eta^{\prime} K^{0}$ & $56.6_{-3.5-3.2}^{+3.6+3.2}$ & - & - \\
$\omega K^{-}$ & $8.1 \pm 0.6 \pm 0.5$ & - & $0.05 \pm 0.08 \pm 0.01$ \\
$\omega \pi^{-}$ & $7.0 \pm 0.6 \pm 0.5$ & - & $-0.03 \pm 0.09 \pm 0.02$ \\
$\omega K^{0}$ & $3.9 \pm 0.7 \pm 0.4$ & - & - \\
$\omega \pi^{0}$ & $0.8_{-0.4}^{+0.5} \pm 0.1$ & $<1.5$ & - \\
$\eta K^{+} \pi^{-}$ & $31.7 \pm 1.9_{-2.6}^{+2.2}$ & - & - \\
$\eta \pi^{+} \pi^{-}$ & $6.2_{-1.6-0.6}^{+1.8+0.8}$ & $<12$ & - \\
$a_{0}^{-} K^{+}$ & - & $<1.6$ & - \\
$a_{0}^{-} \pi^{+}$ & - & $<2.8$ & - \\
\hline
\end{tabular}




\section{Acknowledgments}

The author wish to thank the KEKB accelerator group for the excellent operation of the KEKB accelerator. This work is supported by the National Science Council of the Republic of China under the grant NSC-93-2112-M-002-045.

\section{References}

[1] For a detailed description about theoretical predictions and experimental findings, see K. Abe et al. (Belle Collaboration), BELLE-CONF-0525, hep-ex/0508030 and references therein.

[2] For more details, see K. Abe et al. (Belle Collaboration), BELLE-CONF-0563, hep-ex/0509016.

[3] For more details, see K. Abe et al. (Belle Collaboration), BELLE-CONF-0526, hep-ex/0508052.

[4] K. Abe et al. (Belle Collaboration), BELLE-CONF-0533, hep-ex/0509003.

[5] A. Abashian et al. (Belle Collaboration), Nucl. Instr. and Meth. A 479, 117 (2002).

[6] R. Brun et al., GEANT 3.21, CERN Report No. DD/EE/84-1, 1987.

[7] J. Conrad et al., Phys. Rev. D 67, 012002 (2003).
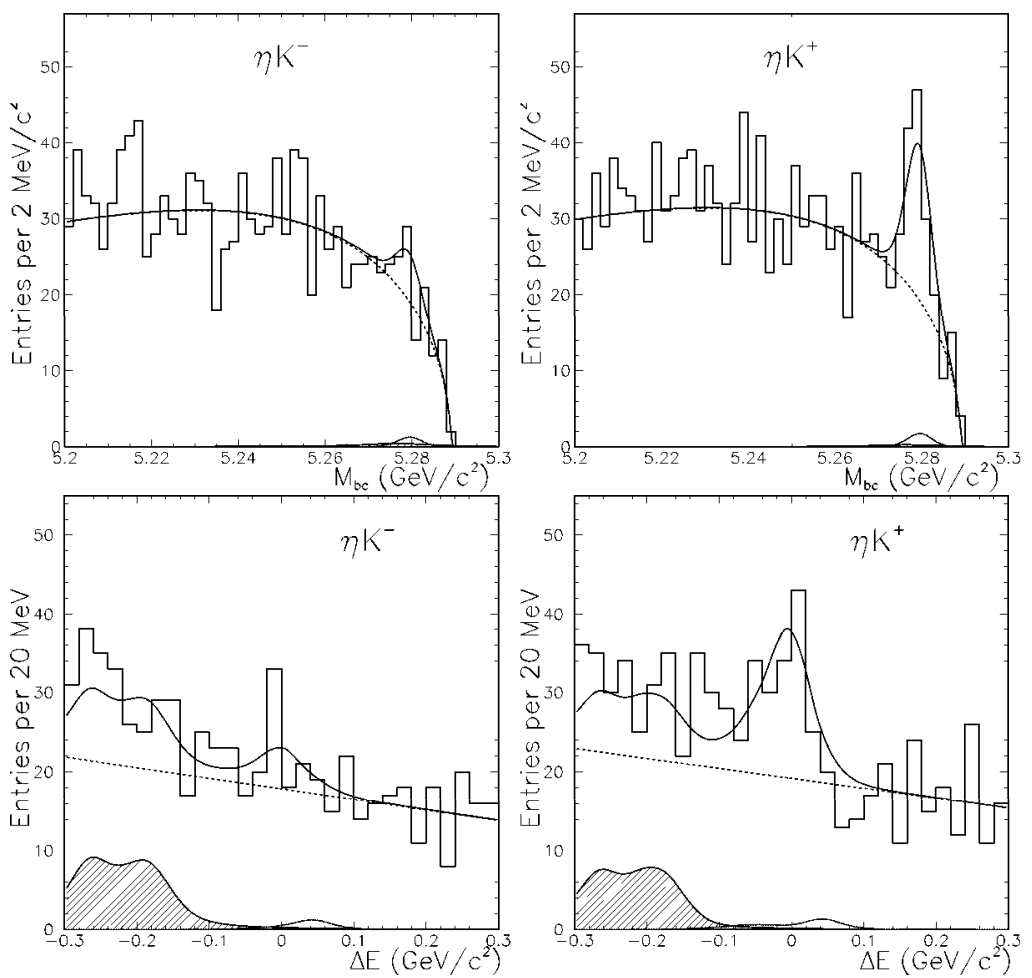

Figure 1: $M_{b c}$ and $\Delta E$ projections for (left) $B^{-} \rightarrow \eta K^{-}$and (right) $B^{+} \rightarrow \eta K^{+}$with the $\eta_{\gamma \gamma}$ and $\eta_{3 \pi}$ modes combined. Open histograms are data, solid curves are the fit functions, dashed lines show the continuum contributions and shaded areas are the contributions from charmless $B$ decays. The small bumps near $M_{b c}=$ $5.28 \mathrm{GeV} / c^{2}$ and $\Delta E=0.05 \mathrm{GeV}$ are the backgrounds from $B^{ \pm} \rightarrow \eta \pi^{ \pm}$due to $\pi-K$ misidentification. 